\title{
DETERMINACIÓN DE LA FAJA MARGINAL DEL RIO CAPLINA EN EL DISTRITO DE PACHIA Y CALANA MEDIANTE MODELAMIENTO HIDRÁULICO
}

\author{
DETERMINATION OF THE MARGINAL SASH OF THE CAPLINA RIVER IN PACHIA AND \\ CALANA DISTRICT BY HYDRAULIC MODELING
}

Información del artículo:

Recibido: 15/10/2020

Aceptado: $25 / 11 / 2020$

Daysi Rossmery Mamani Ccantuta ${ }^{2}$ Alexander Nicolás Vilcanqui Alarcón ${ }^{3}$

Aceptado: $2511 / 2020$

1,2 Escuela de Ingeniera Civil Universidad Privada de Tacna, Perú

${ }^{3}$ Docente escuela de Ingeniera Civil de la Universidad Privada de Tacna, Perú E-mail: ${ }^{1}$ choque95rh@gmail.com, ${ }^{2}$ day.ccantuta@gmail.com, ${ }^{3}$ nicolas_2030@hotmail.com

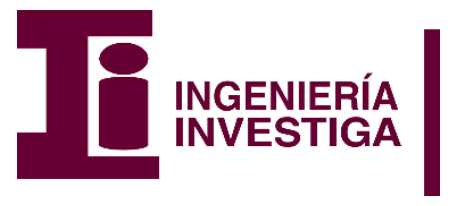

Vol. 2, $\mathrm{N}^{\circ} 2$

julio - diciembre del 2020

ISSN - Online: 2708-3039

DOI: https://doi.org/10.47796/ing.v2i2.413 


\section{Resumen}

En el departamento de Tacna, específicamente los Distritos de Pachía y Calana presentan vulnerabilidad a inundación por máximas avenidas, según las evaluaciones realizadas por La Autoridad Nacional del Agua, en los años del 2001 y 2019 según el registro de datos hidrométricos de la Estación Calientes, se reportaron caudales de $33 \mathrm{~m} 3 / \mathrm{s}$ y $26 \mathrm{~m} 3 / \mathrm{s}$ respectivamente, ocasionando destrucción de las bocatomas de Challata y Calientes, inundación de áreas agrícolas, dejando en aniego la zona de los Baños termales en Calientes, colmatación de las obras de encauzamiento del rio Caplina desde Calana hasta el sector Piedra Blanca. La determinación de una faja marginal mediante un modelamiento hidráulico busca salvaguardar la vida de los habitantes y también los aspectos económicos y ambientales.

Palabras Claves: Inundación; Máximas Avenidas; Modelamiento Hidráulico; Riesgo, Vulnerabilidad.

\section{Abstract}

In the department of Tacna specifically, the Districts of Pachía and Calana show vulnerability to flooding by maximum avenues, according to the evaluations carried out by the National Water Authority, in the years 2001 and 2019 according to the hydrometric data registry of the Calientes Station, reported flows of $33 \mathrm{~m} 3 / \mathrm{s}$ and $26 \mathrm{~m} 3$ / s respectively causing destruction of the intakes of Challata and Calientes, flooding of agricultural areas, leaving the area of the thermal baths in Calientes in flood, clogging of the channeling works of the Caplina river from Calana to the Piedra Blanca sector. The determination of a marginal strip through hydraulic modeling seeks to safeguard the lives of the inhabitants and also the economic and environmental aspects.

Keywords: Flood: Maximum Floods; Hydraulic Modeling; Risk; Vulnerability. 


\section{Introducción}

El crecimiento demográfico desmesurado y desordenado de la sociedad, han establecido su desarrollo económico en los ámbitos colindantes a los cuerpos de agua, cuya expansión demográfica sobre las riberas de los cuerpos de agua se ha traducido en alteraciones cada vez más evidentes en los entornos fluviales. Los cuerpos de agua más afectados son los ríos y su entorno, debido a las intensas lluvias durante las épocas de diciembre, enero, febrero y marzo, las precipitaciones son constantes, incrementando el caudal de los ríos en todos sus niveles: rural, urbano, industrial y agrícola; por lo que cada vez se ejecutan muchas infraestructuras hidráulicas de adecuación para que los ríos se acomoden a las exigencias de la humanidad, que incluyen cambios geomorfológicos como modificaciones en perfiles longitudinales y transversales del cauce del rio. El imprudente asentamiento territorial a las riberas de un rio y la falta de información sobre la conducta fluvial, con llevan a realizar actividades de mitigación en los cauces como son la limpieza, descolmatación y encauzamientos de ríos y quebradas, muchas veces asociados a un estrechamiento excesivo del ancho mínimo de cauce, el resultado puede ser la erosión y desborde del rio provocando inundaciones.

Al respecto la cuenca del Rio Caplina nace en la cordillera del Barroso ubicado a $5300 \mathrm{msnm}$, y discurre sus aguas a través del valle de Tacna, continuando su recorrido por los distritos de Pachia y Calana, para finalmente concluir su recorrido en el océano pacífico luego de atravesar el abanico fluvial de La Yarada. La cuenca Caplina tiene la forma de un cuerpo alargado, estrechándose a medida que el río se acerca al Océano Pacífico y se encuentra ubicado geográficamente entre los paralelos $17^{\circ} 59^{\prime}$ y $18^{\circ}$ $32^{\prime}$ de latitud Sur y los meridianos $69^{\circ} 78^{\prime}$ y $70^{\circ} 48^{\prime}$ de longitud Oeste de Greenwich. Además, La cuenca del río Caplina tiene un área total de drenaje hasta su desembocadura en el océano pacifico de 908,90 $\mathrm{km} 2$, contando con una longitud de cauce principal desde sus nacientes de $87,52 \mathrm{~km}$ y una pendiente media de cauce de 2,86 \%, al respecto diversos estudios (Pino, 2013; Pastrana, 2011); Vilcanqui, 2016) han sido realizados con la finalidad de conocer sus características y modelamientos diversos, que son necesarios de complementar. Por ello es importante hacer la delimitación de fajas marginales para proteger de los recursos hídricos y su entorno que se puede realizar de dos maneras según R.J N³32, 2016.

\section{Objetivos}

Determinar la faja marginal del rio Caplina en los distritos de Pachía y Calana mediante un modelamiento hidráulico.

\section{Metodología}

El tipo de investigación es explicativo ya que se busca explicar el comportamiento de las variables dependientes en función de las independientes para poder explicar, comprender e interpretar 
porque ocurre un fenómeno y en qué condiciones. La recolección de datos es retrospectivo, debido a que los datos se obtienen de fuentes secundarias existentes, como el registro hidrométrico de caudales de los últimos 20 años del Rio Caplina, cuyos datos se encuentran en la base del Sistema Nacional de Información de Recursos Hídricos (SNIRH) de la Autoridad Nacional del Agua (ANA).

\section{Modelamiento Hidrológico}

El modelamiento hidrológico se realizó con fines de representar los procesos hidrológicos de forma simplificada, almacenamientos y flujos del agua en varias direcciones para realizar las predicciones cuantitativamente y cualitativamente, para tal fin se aplicó el modelo HEC-HMS en su diagramación que permití obtener una caracterización hidrológica de la cuenca Caplina. Para el modelamiento hidrológico de la cuenca del río Caplina se empleó los datos Hidrométricos del registro histórico de caudales máximos diarios de los últimos 20 años de la estación Calientes ubicado en Coordenadas UTM Datum WGS84 zona 19k 381103E; 8025356N que se encuentran en la base del Sistema Nacional de Información de Recursos Hídricos (SNIRH) de la Autoridad Nacional del Agua (ANA). En la Figura 1 se observa la Delimitación de la Cuenca Caplina aplicando la herramienta HEC-HMS.

\section{Figura 1}

Delimitación de la cuenca Caplina aplicando la herramienta de HEC - HMS

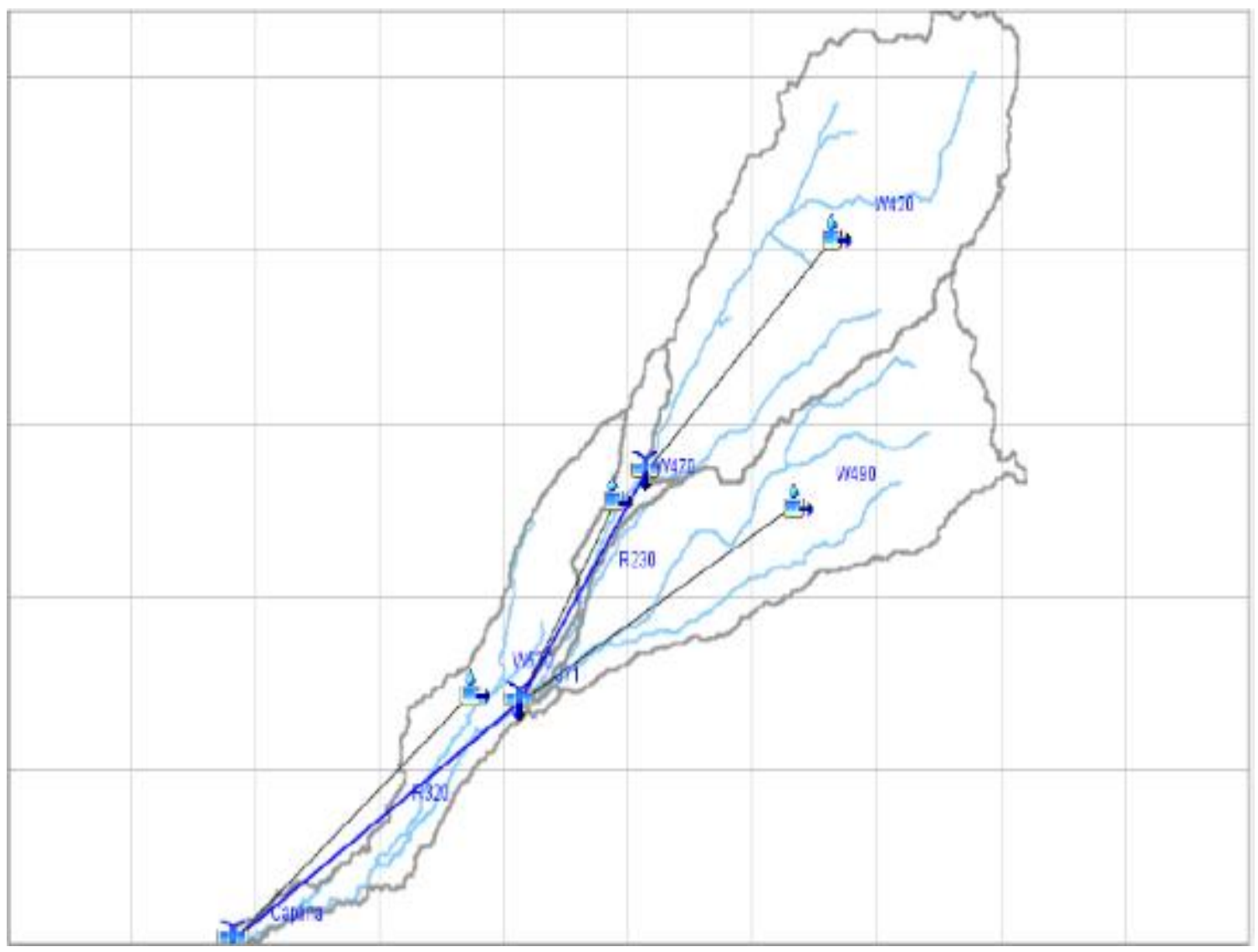

Nota. Elaboración propia. 
Con la información se realizó la simulación de los procesos de precipitación-escorrentía y posteriormente obtener los hidrogramas de las máximas avenidas para un tiempo de retorno (TR) de 50 y 100 años. En la figura 2 se observa el Hidrograma de Máximas Avenidas para un TR de 50 años, mientras en la figura 3 se observa el Hidrograma de Máximas Avenidas para un TR de 100 años de la cuenca del rio Caplina.

\section{Figura 2}

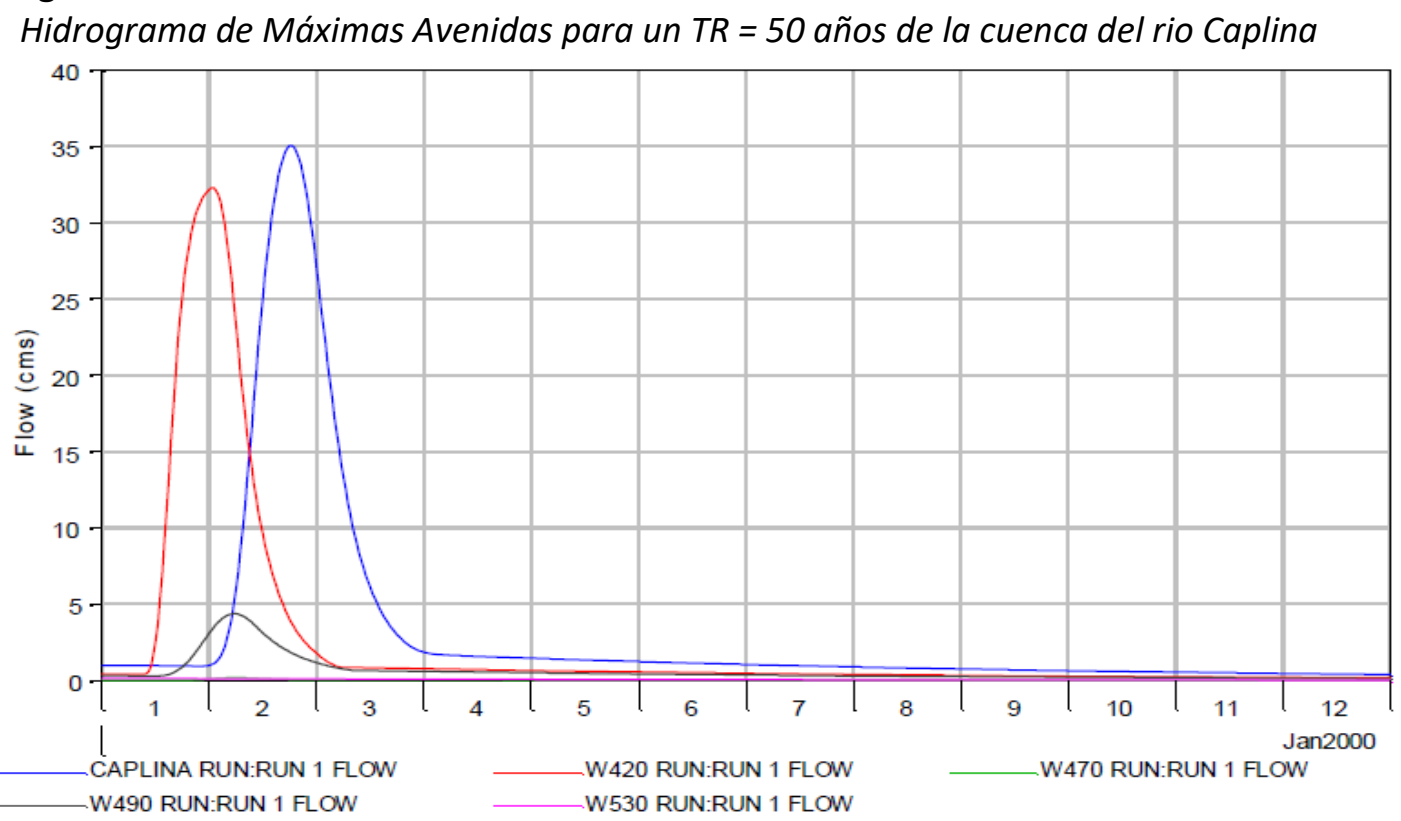

Nota. Elaboración propia.

Figura 3

Hidrograma de Máximas Avenidas para un TR = 100 años de la cuenca del rio Caplina

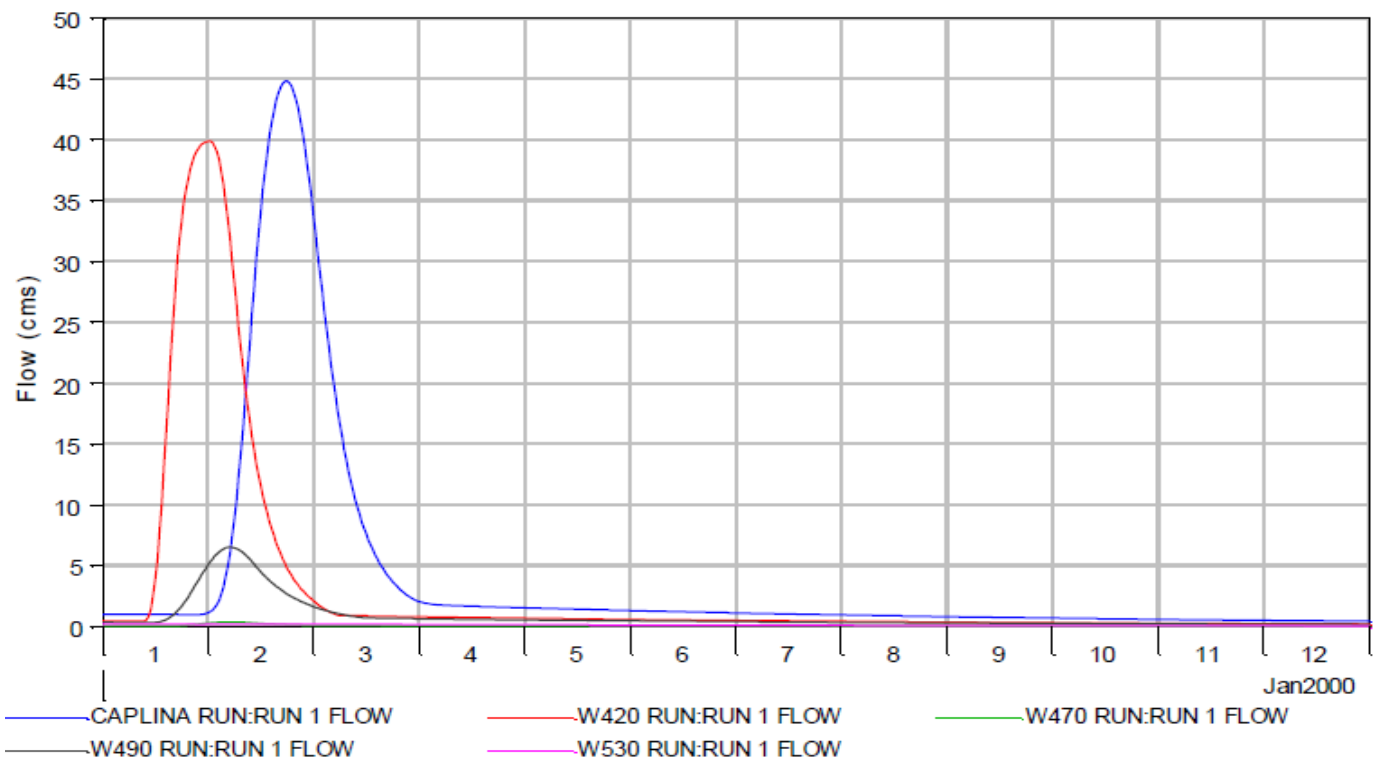

Nota. Elaboración propia. 
En la Tabla 1 se muestra los datos hidrométricos del registro histórico de caudales de los últimos 20 años del rio Caplina y la quebrada Palca, en el caso de este último se generó empíricamente los datos hidrométricos de mediante el modelo Autorregresivo AR (1), también conocido como Modelo Markoviano de primer orden, para ello se determinó los parámetros estadísticos y realizar las distribuciones estadísticas para verificar si los datos hidrométricos obtenidos de la estación Calientes del Rio Caplina y los datos obtenidos empíricamente se ajustan a una distribuciones estadísticas (normal, Log normal, gama, Log de Pearson, etc) .

Tabla 1

Registro histórico de Caudales de Máximas Avenidas

\begin{tabular}{|c|c|c|}
\hline Año & $\begin{array}{l}\text { Rio Caplina } \\
\text { (m3/s) }\end{array}$ & $\begin{array}{c}\text { Quebrada Palca } \\
(\mathrm{m} 3 / \mathrm{s})\end{array}$ \\
\hline 2000 & 6,89 & 0,52 \\
\hline 2001 & 33,22 & 0,74 \\
\hline 2002 & 5,20 & 0,44 \\
\hline 2003 & 1,49 & 0,52 \\
\hline 2004 & 3,21 & 0,17 \\
\hline 2005 & 2,94 & 1,45 \\
\hline 2006 & 4,07 & 0,18 \\
\hline 2007 & 2,41 & 1,61 \\
\hline 2008 & 4,57 & 1,11 \\
\hline 2009 & 3,65 & 0,53 \\
\hline 2010 & 2,71 & 0,90 \\
\hline 2011 & 4,30 & 0,96 \\
\hline 2012 & 11,63 & 0,20 \\
\hline 2013 & 6,00 & 0,74 \\
\hline 2014 & 5,55 & 1,01 \\
\hline 2015 & 2,68 & 0,75 \\
\hline 2016 & 1,45 & 1,02 \\
\hline 2017 & 1,68 & 0,65 \\
\hline 2018 & 1,68 & 0,28 \\
\hline 2019 & 26,28 & 0,30 \\
\hline
\end{tabular}

Nota. Elaboración propia.

A través de HIDROESTA 2.0 se realizó los cálculos hidrológicos y estadísticos que se muestran en la tabla 2 y 3. 
Tabla 2

Distribuciones Estadísticas para la estimación de caudales de diseño - rio Caplina

\begin{tabular}{|c|c|c|c|c|c|c|c|c|}
\hline \multirow[b]{2}{*}{ Distribuciones } & \multicolumn{2}{|c|}{$\Delta$} & \multicolumn{6}{|c|}{ Caudales máximos diarios $\left(\mathrm{m}^{3} / \mathrm{s}\right)$} \\
\hline & $\begin{array}{l}\text { Delta } \\
\text { teórico }\end{array}$ & $\begin{array}{c}\text { Delta } \\
\text { tabular }\end{array}$ & TR2 & $\begin{array}{c}\text { TR } \\
5 \\
\end{array}$ & $\begin{array}{l}\text { TR } \\
10 \\
\end{array}$ & $\begin{array}{l}\text { TR } \\
25 \\
\end{array}$ & $\begin{array}{l}\text { TR } \\
50 \\
\end{array}$ & $\begin{array}{c}\text { TR } \\
100 \\
\end{array}$ \\
\hline Distribución normal & 0,2257 & 0,1741 & 5,83 & 10,81 & 13,41 & 16,19 & 17,98 & 19,59 \\
\hline $\begin{array}{l}\text { Distribución log Normal } 2 \\
\text { parámetros }\end{array}$ & 0,0672 & 0,1741 & 4,23 & 8,07 & 11,32 & 16,24 & 20,51 & 25,29 \\
\hline $\begin{array}{l}\text { Distribución log normal } 3 \\
\text { parámetros }\end{array}$ & 0,0617 & 0,1741 & 4,17 & 8,02 & 11,34 & 16,42 & 20,88 & 25,93 \\
\hline $\begin{array}{l}\text { Distribución Gamma de } 2 \\
\text { parámetros }\end{array}$ & 0,1283 & 0,1741 & 4,74 & 8,9 & 11,79 & 15,46 & 18,16 & 20,81 \\
\hline $\begin{array}{l}\text { Distribución log Pearson } \\
\text { tipo III }\end{array}$ & 0,0439 & 0,1741 & 4 & 7,9 & 11,65 & 18,09 & 24,37 & 32,17 \\
\hline Distribución Gumbel & 0,2012 & 0,1741 & 4,86 & 10,09 & 13,55 & 17,92 & 21,16 & 24,38 \\
\hline Distribución log Gumbel & 0,0753 & 0,1741 & 3,72 & 7,35 & 11,52 & 20,35 & 31,02 & 47,14 \\
\hline
\end{tabular}

Nota. Elaboración propia.

Tabla 3

Distribuciones Estadísticas para la estimación de caudales de diseño-quebrada Palca

\begin{tabular}{cccccccccc}
\hline & \multicolumn{3}{c}{$\boldsymbol{\Delta}$} & \multicolumn{5}{c}{ Caudales máximos diarios $\left(\mathbf{m}^{\mathbf{3}} / \mathbf{s}\right)$} \\
\cline { 2 - 8 } Distribuciones & $\begin{array}{c}\text { Delta } \\
\text { teórico }\end{array}$ & $\begin{array}{c}\text { Delta } \\
\text { tabular }\end{array}$ & $\begin{array}{c}\text { TR } \\
\mathbf{2}\end{array}$ & $\begin{array}{c}\text { TR } \\
\mathbf{5}\end{array}$ & $\begin{array}{c}\text { TR } \\
\mathbf{1 0}\end{array}$ & $\mathbf{2 5}$ & $\mathbf{5 0}$ & $\mathbf{1 0 0}$ \\
\hline $\begin{array}{c}\text { Distribución normal } \\
\text { Distribución log normal 2 } \\
\text { parámetros }\end{array}$ & 0,0769 & 0,1741 & 0,85 & 1,33 & 1,58 & 1,85 & 2,02 & 2,18 \\
$\begin{array}{c}\text { Distribución log normal 3 } \\
\text { parámetros }\end{array}$ & 0,1135 & 0,1741 & 0,63 & 1,33 & 1,97 & 3,00 & 3,93 & 5,01 \\
$\begin{array}{c}\text { Distribución Gamma de 2 } \\
\text { parámetros }\end{array}$ & 0,0655 & 0,1741 & 0,74 & 1,27 & 1,63 & 2,09 & 2,44 & 2,79 \\
$\begin{array}{c}\text { Distribución Gamma de 3 } \\
\text { parámetros }\end{array}$ & 0,0674 & 0,1741 & 0,79 & 1,30 & 1,61 & 1,96 & 2,21 & 2,44 \\
$\begin{array}{c}\text { Distribución Gumbel } \\
\text { Distribución log Gumbel }\end{array}$ & 0,0721 & 0,1828 & 0,1741 & 0,76 & 1,26 & 1,59 & 2,01 & 2,33 & 2,64 \\
\hline
\end{tabular}

Nota. Elaboración propia.

En la tabla 2 y 3 se determinó los caudales de diseño en $\mathrm{m}^{3} / \mathrm{s}$ con la distribución estadística aceptando que la distribución Log Pearson tipo III se ajusta a la serie histórica de caudales al poseer el menor valor del delta teórico, siendo necesario determinar los caudales máximos instantáneos aplicando la fórmula de Fuller [1]. 


$$
\begin{aligned}
& \operatorname{Qmax}_{\text {inst }}=C * Q m d \\
& C=1+\frac{2.66}{A^{0,3}}
\end{aligned}
$$

Donde:

Qmd : Caudal máximo diario $\left(\mathrm{m}^{3} / \mathrm{s}\right)$

C : Coeficiente de ajuste

A : Área drenada de la cuenca

Los resultados de la estimación de los caudales instantáneos se muestran en la tabla 4.

Tabla 4

Estimación de caudales máximos instantáneos $\left(\mathrm{m}^{3} / \mathrm{s}\right)$ para modelamiento hidráulico según el tiempo de

\begin{tabular}{|c|c|c|c|c|c|c|}
\hline \multirow[b]{2}{*}{ Descripción } & \multicolumn{6}{|c|}{$\begin{array}{c}\text { Tiempo de retorno (TR) } \\
\text { ( años ) }\end{array}$} \\
\hline & $\mathrm{TR}=\mathbf{2}$ & $\mathrm{TR}=5$ & $T R=10$ & $T R=25$ & $\mathrm{TR}=\mathbf{5 0}$ & $\mathrm{TR}=100$ \\
\hline Caudal de diseño $\left(\mathrm{m}^{3} / \mathrm{s}\right)$ & 4,74 & 9,17 & 13,28 & 20,18 & 26,81 & 34,96 \\
\hline Caudal máximo Instantáneo $\left(\mathrm{m}^{3} / \mathrm{s}\right)$ & 6,37 & 12,33 & 17,86 & 27,13 & 36,05 & 47,01 \\
\hline
\end{tabular}
retorno en años

Nota. Elaboración propia.

\section{Modelamiento Hidráulico}

El modelamiento Hidráulico busca simular el flujo del agua sobre la superficie topográfica, siguiendo la premisa de que el agua, escurre a favor de la gravedad. Para la visualización del desplazamiento de la escorrentía superficial por gravedad se ha aplicado el software HEC RAS v. 5.0.7 que está basado en la resolución de la ecuación de la energía unidimensional, donde las pérdidas de energía fueron evaluadas por fricción y contracción u expansión, de esta manera se demuestra si el caudal máximo instantáneo estimado supera el tirante de agua del cauce o no para un periodo de retorno de $2,5,10,25,50$ y 100 años.

\section{Configuración del modelo digital del terreno}

La configuración del modelo digital del terreno es un conjunto de procedimientos, uso de herramientas y utilidades especialmente diseñadas para procesar datos topográficos georreferenciados en el sistema de coordenadas UTM que nos permiten complementar al modelamiento hidráulico con Hec Ras v 5.0.7 bajo el entorno del sistema de información geográfica (SIG) y la representación visual, matemática de los valores de la cota de elevación con respecto al nivel medio del mar en un tramo total de 1,5 km, la representación para la cuenca Caplina se observa en la figura 4. 


\section{Figura 4}

Representación gráfica del modelo digital del terreno

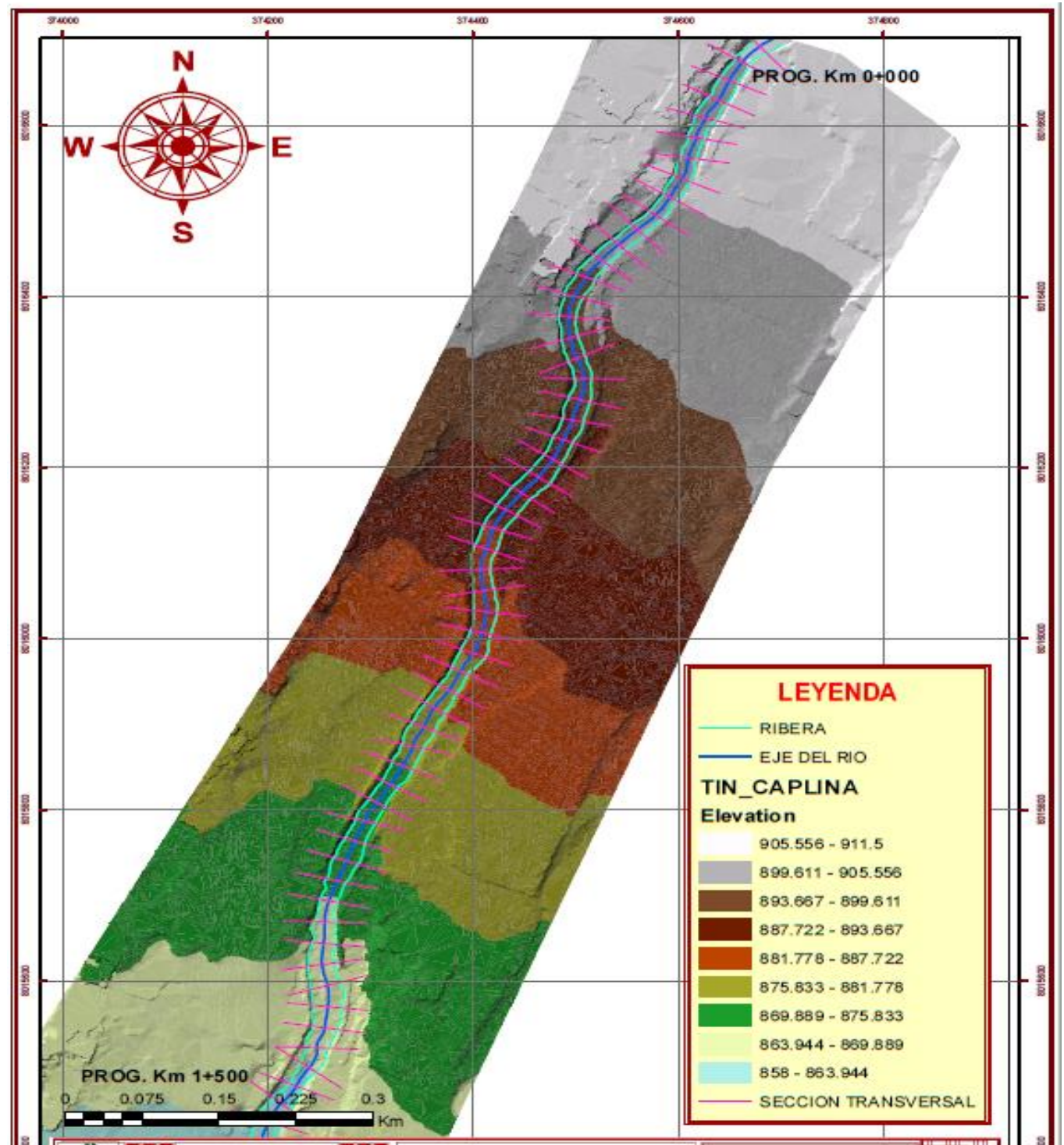

Nota. Modelamiento hidráulico realizado con Hec Ras v 5.0.7

\section{Configuración de la geométrica del cauce y Parámetros Hidráulicos}

La configuración de la geometría del cauce consiste en definir los elementos de un cauce natural, para ello se ha aplicado la extensión Hec-GeoRas del software ArcGis 10.5 de tal modo se define el ancho del cauce natural y el límite superior de la ribera del rio. Mientras que la configuración de parámetros hidráulicos consiste en definir el tipo de flujo del cauce natural de ríos y quebradas, para el modelamiento hidráulico del Rio Caplina en una longitud de $1,5 \mathrm{Km}$ se ha considerado un flujo no permanente ya que la velocidad de escurrimiento varía con el tiempo. Además de acuerdo a las bases teóricas y ecuaciones Hidráulicas se determinó un valor del coeficiente de Rugosidad Manning de 0,035 
y una pendiente de la zona de investigación de 3,16\%. Asimismo se usó el software HecRas para trabajar los datos de Caudales máximos Instantáneo estimados para un tiempo de retorno de 50 y 100 años y finalmente se realizó el procesamiento computacional de la información alimentada en el software Hec Ras v. 5.0,7.

\section{Resultados}

\section{Escenario para tiempo de retorno de 2, 5,10 y 25 años}

Mediante el modelamiento hidráulico considerando un escenario de tiempo de retorno de 2, 5, 10 y 25 años, simulado con un caudal máximo instantáneo de $6,37 \mathrm{m3} / \mathrm{s}, 12,33 \mathrm{m3} / \mathrm{s}, 17,86 \mathrm{~m} 3 / \mathrm{s}$ y $27,13 \mathrm{~m} 3 / \mathrm{s}$ respectivamente se determinó un área total de inundación 267,09 m2 en ambas márgenes del rio Caplina en los distrito de Calana y Pachía, ubicado en la progresiva $\mathrm{Km} 1+475 \mathrm{~m}$, cuyo tirante máximo de flujo alcanzado para este escenario fue de $1,17 \mathrm{~m}$ y una velocidad máxima $4,22 \mathrm{~m} / \mathrm{s}$ obtenido del procesamiento computacional a través del Sistema de Información Geográfica (SIG), tal como se puede apreciar en la figura 5.

\section{Figura 5}

Sección transversal de la progresiva $\mathrm{Km} 0+025 \mathrm{~m}$ (flujo supercrítico)

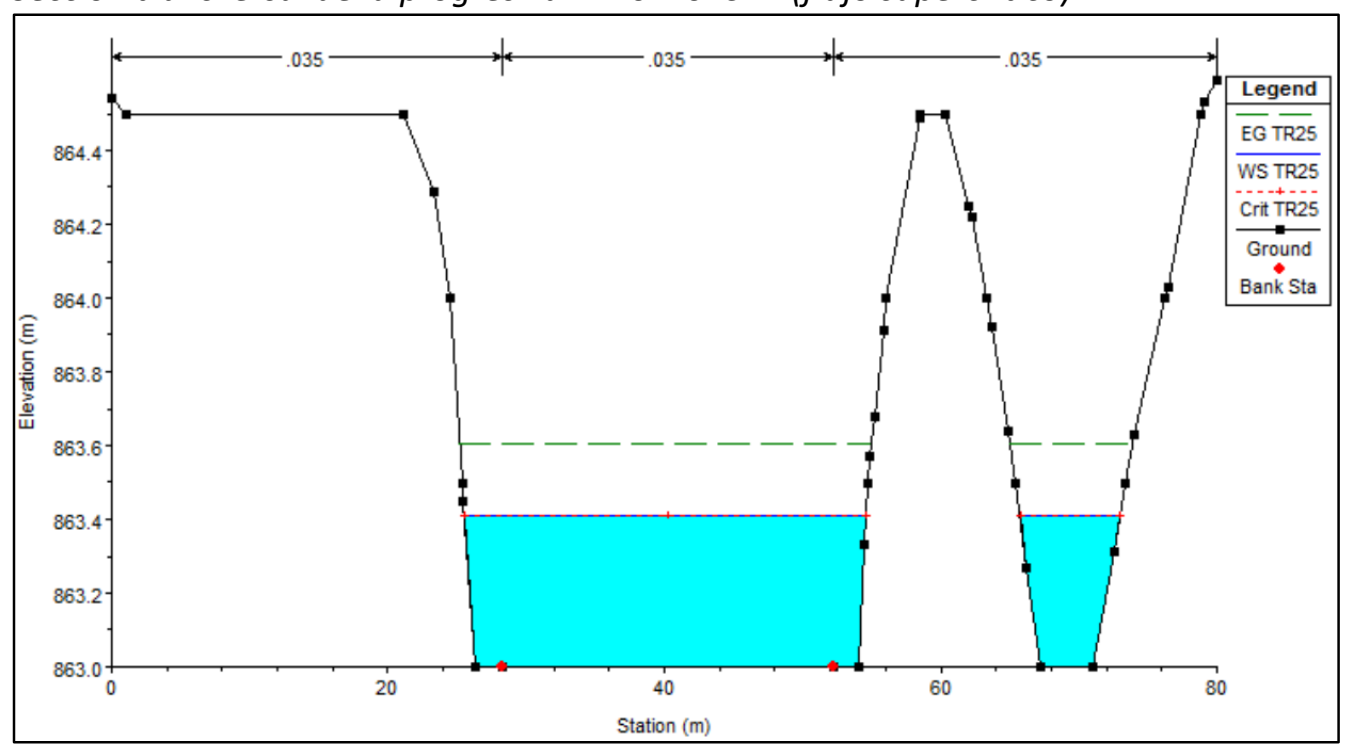

Nota: elaboración propia aplicando el software Hec-Ras Vs5.0.7 


\section{Escenario para tiempo de retorno de 50 Años}

Mediante el modelamiento hidráulico considerando un escenario de tiempo de retorno de 50 años, simulado con un caudal máximo instantáneo de $36,05 \mathrm{~m}^{3} / \mathrm{s}$ se determinó un área total de inundación 388,25 $\mathrm{m}^{2}$ en ambas márgenes del rio Caplina en los distrito de Calana y Pachía, ubicado en las progresiva $\mathrm{Km} 0+550 \mathrm{~m}$ y $\mathrm{Km} 1+475 \mathrm{~m}$, cuyo tirante máximo de flujo alcanzado para este escenario fue de 1,33 $\mathrm{m}$ y una velocidad máxima $4,55 \mathrm{~m} / \mathrm{s}$ obtenido del procesamiento computacional a través del Sistema de Información Geográfica (SIG), tal como se puede apreciar en la figura 6 y figura 7.

\section{Figura 6}

Sección transversal de la progresiva Km $0+550 m$ (flujo supercrítico $N F=1,58$ )

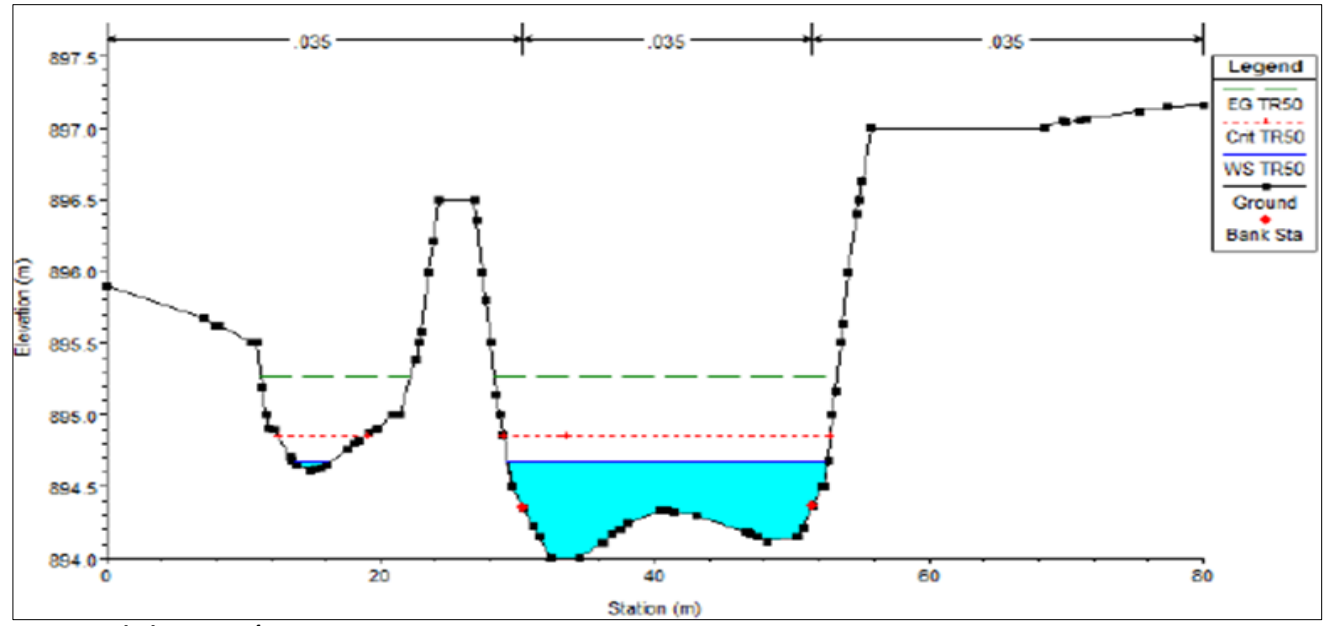

Nota. Elaboración propia.

\section{Figura 7}

Sección transversal de la progresiva Km $1+475 m$ (flujo supercrítico NF=1,02)

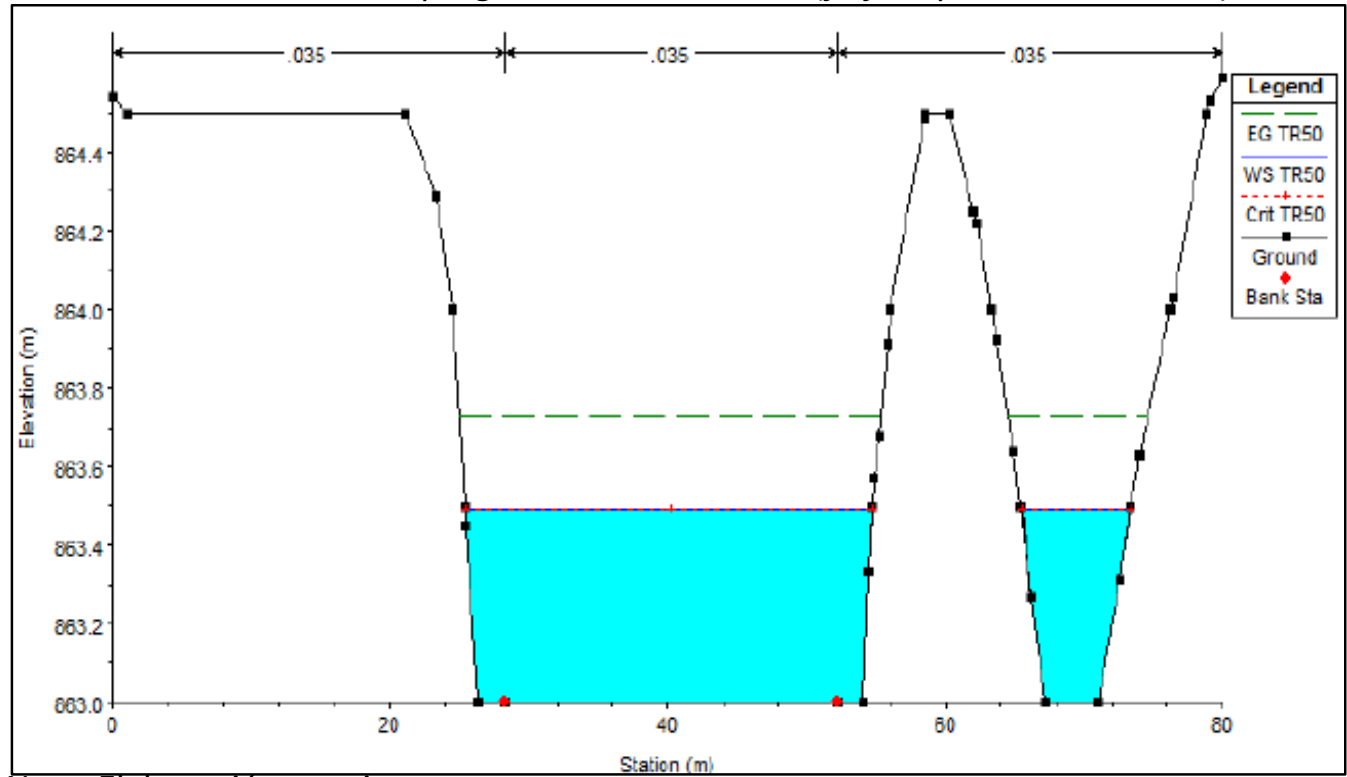

Nota. Elaboración propia. 


\section{Escenario para tiempo de retorno de 100 Años}

El modelamiento para un escenario de tiempo de retorno 100 años, simulado con un caudal máximo instantáneo de 47,01 m³ mostró que el área total de inundación $673,83 \mathrm{~m}^{2}$ en ambas márgenes del rio Caplina en los distrito de Calana - Pachía, ubicado en las progresiva $\mathrm{Km} 0+625 \mathrm{~m}, \mathrm{Km} 1$ + $300 \mathrm{~m}$, cuyo tirante máximo de flujo alcanzado para este escenario fue de 1,41 m y una velocidad máxima $4,87 \mathrm{~m} / \mathrm{s}$ obtenido del procesamiento computacional a través del Sistema de Información Geográfica (SIG). Por lo tanto, la clasificación de flujo según el número de Froude en esta sección se considera como un flujo Supercrítico al poseer un número de Froude igual a 1,65, según la figura 8 y figura 9.

\section{Figura 8}

Sección transversal de la progresiva $\mathrm{Km} 0+625 \mathrm{~m}$ (flujo supercrítico $N F=1,94 F u e n t e:$ Elaboración propia aplicando el software Hec-Ras Vs5.0.7)

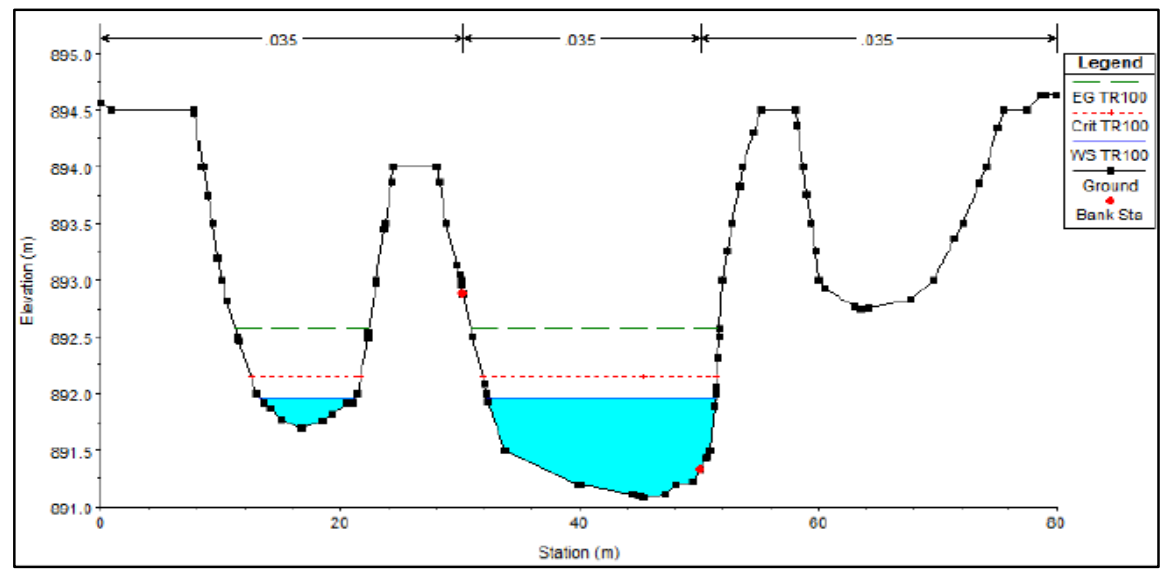

Nota. Elaboración propia.

\section{Figura 9}

Sección transversal de la progresiva Km $1+300$ m (flujo supercrítico NF=1,10)

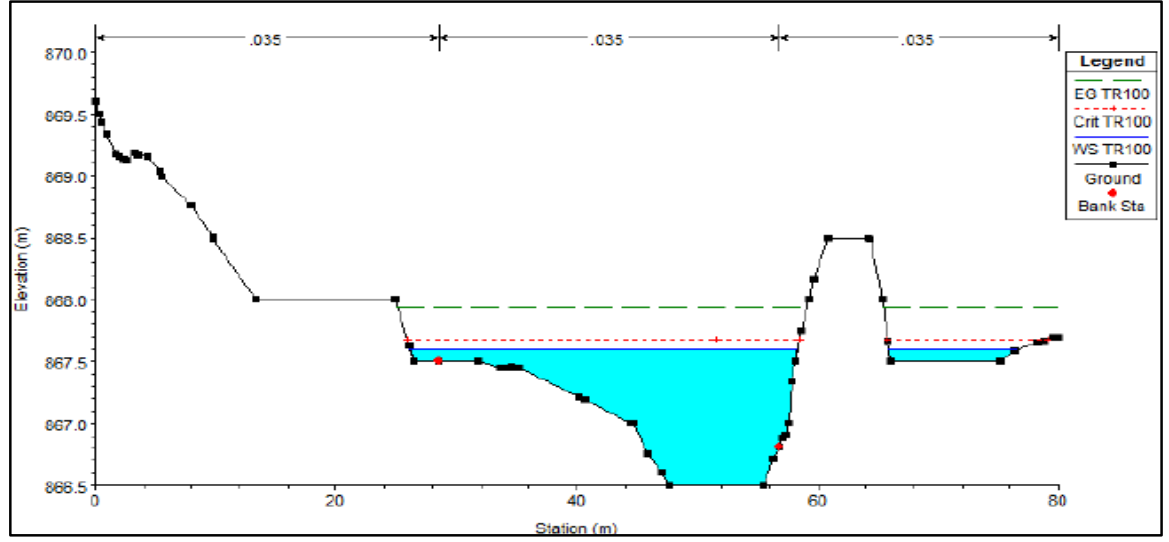

Nota. Elaboración propia. 


\section{Franjas marginales}

La faja marginal es un bien de dominio hidráulico público, y se realiza una delimitación de fajas marginales para proteger los recursos hídricos y su entorno; se puede realizar la delimitación de dos maneras según Resolución Jefatural N³32 - 2016 - ANA, mediante un modelamiento hidráulico o mediante huellas máximas. Para la delimitación de la faja marginal se realizó considerando algunos criterios como es la magnitud e importancia de las estructuras hidráulicas de las presas, reservorios, embalses, canales de derivación, entre otros existentes colindantes al Río Caplina. Además la faja marginal delimita el espacio necesario para la construcción, conservación y protección de las defensas ribereñas y de los cauces (figura 9), para ello en la figura 10 se muestra los mapas de inundación.

\section{Figura 9}

Mapa de profundidad del flujo y velocidad del flujo

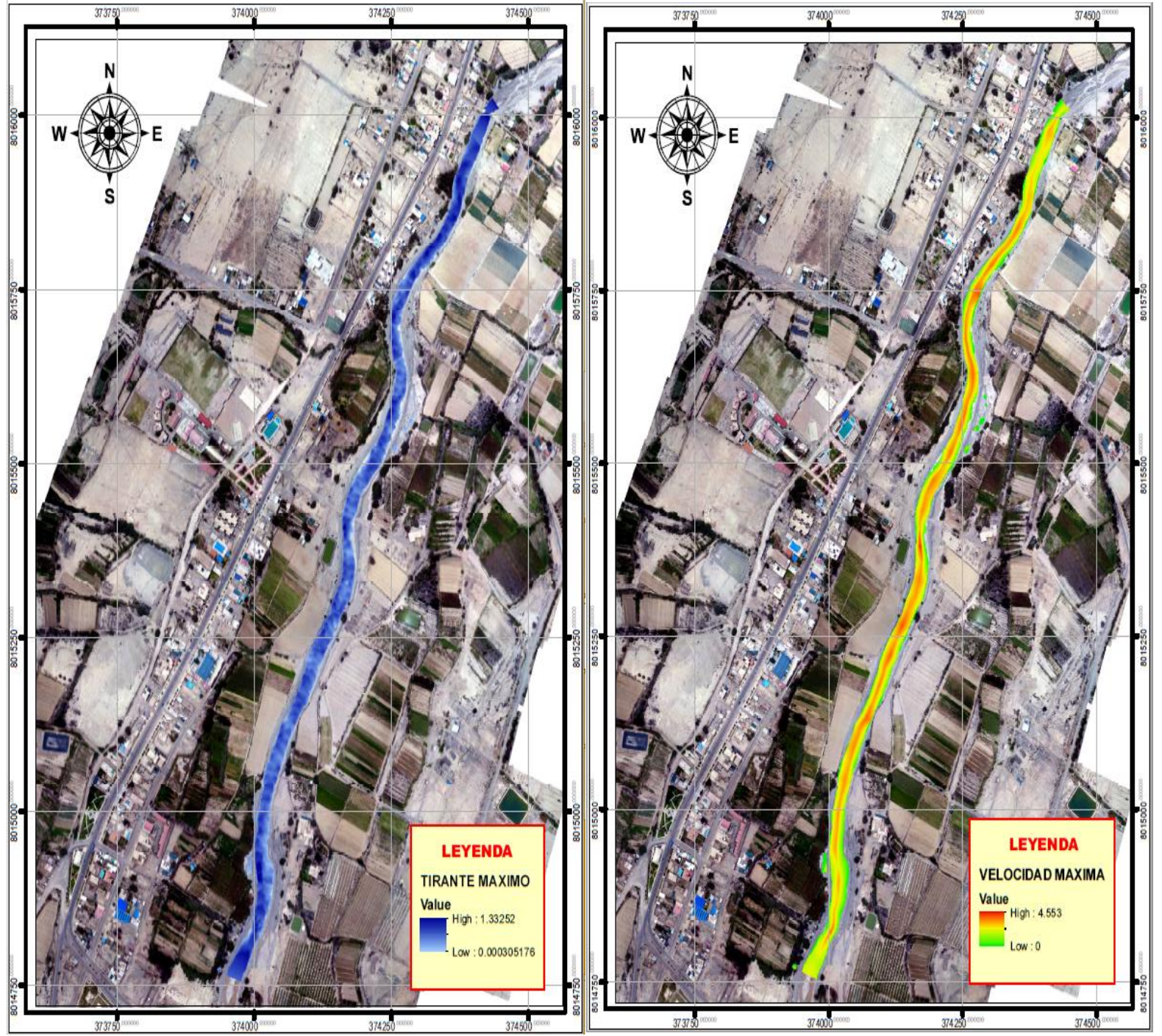

Nota. Elaboración propia, Mapa izquierda profundidad de flujo, Mapa derecha velocidad de flujo. 
Figura 10

Mapa de inundación del flujo

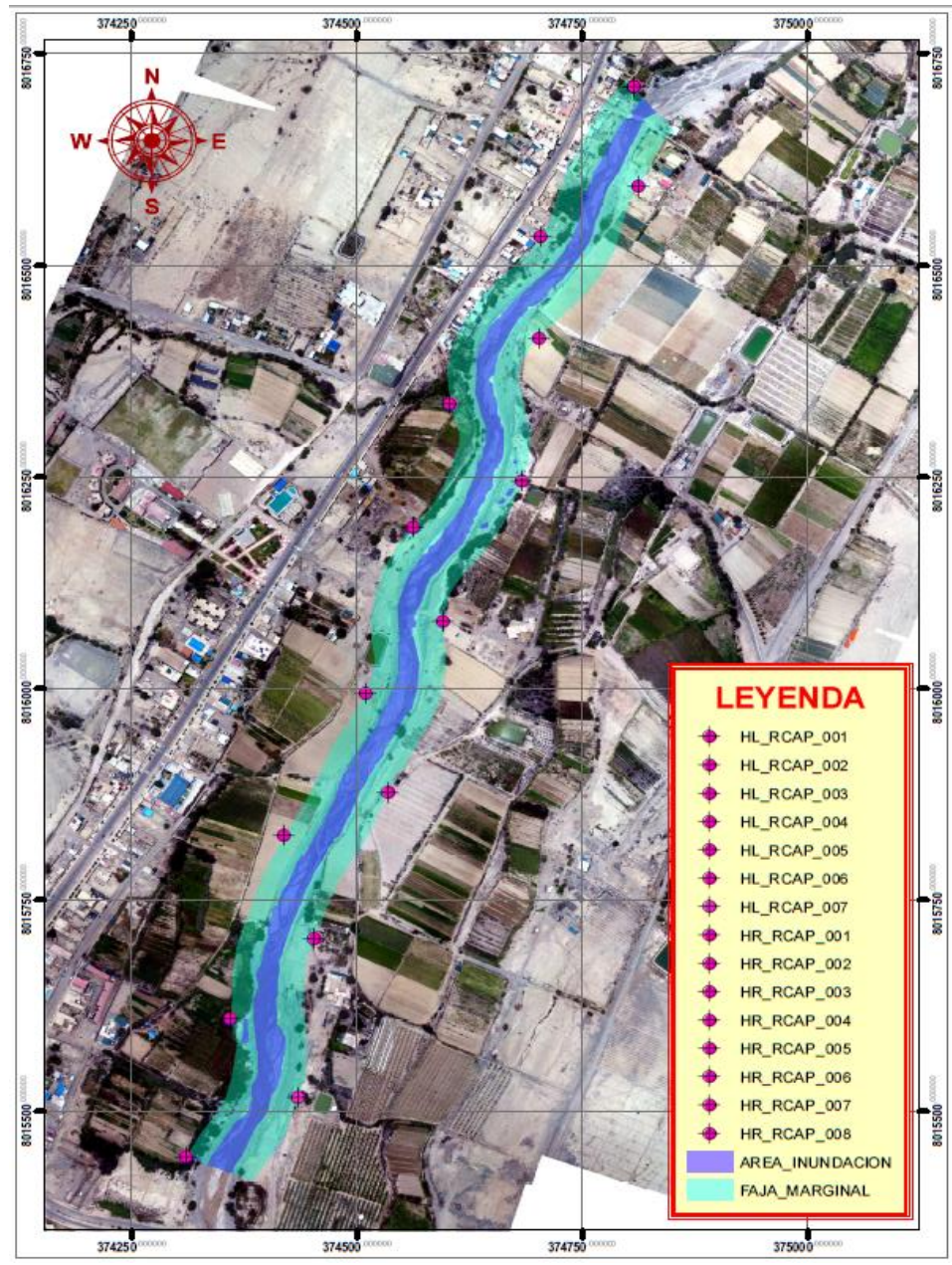

Nota. Elaboración propia.

\section{Discusiones}

Para un escenario con tiempo de retorno de 50 años, de acuerdo a la clasificación de inundaciones según la duración del drenaje de aguas superficiales en base a la matriz de análisis de riesgos del Instituto Nacional de Defensa Civil será de tipo dinámico ya que el producto de la profundidad por la velocidad del flujo resulto 2,06 y su número de Froude fue de 1,76. Por tanto según los niveles de intensidad del Sistema Nacional de Gestión del Riesgo de Desastres (SINAGERD) y el Centro Nacional de Estimación, Prevención y Reducción del Riesgo de Desastres (CENEPRED) se considera nivel de amenaza alto ya que el producto de la velocidad y la profundidad del flujo es mayor igual a 1,5 y según la clasificación de flujos según el número de Froude en esta sección se considera como un flujo supercrítico al poseer un número de Froude mayor a 1, tal como se aprecia en la tabla 5.

Para un escenario con tiempo de retorno de 100 años, de acuerdo a la clasificación de inundaciones según la duración del drenaje de aguas superficiales en base a la matriz de análisis de 
riesgos del Instituto Nacional de Defensa Civil será de tipo dinámico ya que el producto de la profundidad por la velocidad del flujo resulto 6,87 y su número de Froude fue de 1,65. Por tanto según los niveles de intensidad del Sistema Nacional de Gestión del Riesgo de Desastres (SINAGERD) y el Centro Nacional de Estimación, Prevención y Reducción del Riesgo de Desastres (CENEPRED) se considera nivel de amenaza alto ya que el producto de la velocidad y la profundidad del flujo es mayor igual a 1,5 y según la clasificación de flujos según el número de Froude en esta sección se considera como un flujo supercrítico al poseer un número de Froude mayor a 1. La figura 6 muestra la evaluación de la frecuencia, recurrencia o periodo de retorno

\section{Tabla 5}

Niveles o magnitud de intensidad

\begin{tabular}{ccc}
\hline $\begin{array}{c}\text { Niveles de } \\
\text { intensidad }\end{array}$ & Inundaciones estáticas & $\begin{array}{c}\text { Inundaciones } \\
\text { dinámicas }\end{array}$ \\
Muy alta & $\mathrm{H}>1.5 \mathrm{~m}$ & $\mathrm{H}^{*} \mathrm{~V}>1,5 \mathrm{~m}$ \\
Alta & $0,5 \mathrm{~m}<\mathrm{H}<1,5 \mathrm{~m}$ & $0,5 \mathrm{~m}<\mathrm{H}^{*} \mathrm{~V}<1,5 \mathrm{~m}$ \\
Media & $0,25 \mathrm{~m}<\mathrm{H}<0,5 \mathrm{~m}$ & $0,25 \mathrm{~m}<\mathrm{H}^{*} \mathrm{~V}<0,5 \mathrm{~m}$ \\
Baja & $\mathrm{H}<0,25 \mathrm{~m}$ & $\mathrm{H}^{*} \mathrm{~V}<0,25 \mathrm{~m}$ \\
\hline
\end{tabular}

Nota. Elaboración propia.

Tabla 6

Evaluación de la frecuencia, recurrencia o periodo de retorno

\begin{tabular}{cc}
\hline Niveles de intensidad & $\begin{array}{c}\text { Periodo de retorno en años } \\
\text { (TR) }\end{array}$ \\
\hline Muy alta & $1<T<5$ años \\
Alta & $5<T<15$ años \\
Media & $15<T<50$ años \\
Baja & $50<T<200$ años \\
\hline
\end{tabular}

Nota. Elaboración propia.

Tabla 7

Determinación del grado de peligrosidad por inundación

\begin{tabular}{|c|c|c|c|c|c|}
\hline \multirow{4}{*}{ 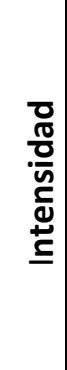 } & Muy Alta & $\begin{array}{c}\text { Peligro Medio } \\
0,25\end{array}$ & $\begin{array}{c}\text { Peligro Alto } \\
0,5\end{array}$ & $\begin{array}{l}\text { Peligro Muy } \\
\text { Alto } 0,75\end{array}$ & $\begin{array}{l}\text { Peligro Muy } \\
\text { Alto } 1\end{array}$ \\
\hline & Alta & $\begin{array}{c}\text { Peligro Bajo } \\
0,19\end{array}$ & $\begin{array}{c}\text { Peligro Medio } \\
0,38\end{array}$ & $\begin{array}{c}\text { Peligro Alto } \\
0,56\end{array}$ & $\begin{array}{c}\text { Peligro Muy } \\
\text { Alto } 0,75\end{array}$ \\
\hline & Media & $\begin{array}{l}\text { Peligro Bajo } \\
0,13\end{array}$ & $\begin{array}{c}\text { Peligro Bajo } \\
0,25\end{array}$ & $\begin{array}{c}\text { Peligro Bajo } \\
0,38\end{array}$ & $\begin{array}{c}\text { Peligro Alto } \\
0,5\end{array}$ \\
\hline & \multirow[t]{3}{*}{ Baja } & $\begin{array}{l}\text { Peligro Bajo } \\
0,06\end{array}$ & $\begin{array}{c}\text { Peligro Bajo } \\
0,13\end{array}$ & $\begin{array}{c}\text { Peligro Bajo } \\
0,19\end{array}$ & $\begin{array}{c}\text { Peligro Medio } \\
0,25\end{array}$ \\
\hline & & Baja & Media & Alta & Muy Alta \\
\hline & & \multicolumn{4}{|c|}{ Frecuencia (Años) } \\
\hline
\end{tabular}

Nota. Elaboración propia. 
Además, a través de la plataforma del Sistema Catastral para predios Rurales del Ministerio de Agricultura y Riego se identificó que las áreas afectadas corresponden a los predios rurales con códigos de Unidad Catastral Nº0625 y Nº0626, en coordenadas UTM Datum WGS84 (8015440 N; 374320 E; $8016240 \mathrm{~N}, 374668 \mathrm{E})$ zona $19 \mathrm{~K}$ respectivamente. Al considerarse esta zona un nivel de amenaza alta ocasionaría graves daños las zonas agrícolas e infraestructura de riego convirtiéndose en una zona no apta para la instalación, expansión de áreas agrícolas o densificación de asentamientos humanos, cuyo predios rurales y viviendas construidas en los márgenes del rio Caplina deberán ser reubicadas a largo plazo con fines de realizar un ordenamiento y planificación territorial, o protegerlas con obras de protección, sistemas de alerta temprana y evacuación temporal.

Para la delimitación de la Faja marginal del Rio Caplina se debe tomo en cuenta la determinación del ancho mínimo de la faja marginal de un cauce ya que en muchos casos, resulta insuficiente frente a los caudales obtenidos mediante el modelamiento hidráulico, ya que los polígonos de inundación sobrepasan su capacidad y es evidente que la mayoría de agricultores, han extendido sus terrenos agrícolas al interior del cauce del rio Caplina, alterando y obstruyendo el transito del flujo de agua que discurre en épocas de avenidas, ocasionando la aparición de tramos sinuosos y desbordes en zonas bajas. Además se ha observado que actualmente parte de la población urbana del el distrito de Pachía se encontraba asentado sobre la rivera del Rio Caplina por lo que se requiere hacer obras de mitigación dado que el flujo en tiempo de avenidas no solo acarrea agua sino además sedimentos, acarrea todo lo que se encuentre dentro del cauce, por tanto es necesario construir defensas ribereñas y defensas vivas a fin de mitigar la socavación de la ribera en su margen derecho ocasionado por las avenidas máximas. según el Art.13 criterios para determinar un ancho mayor de las fajas marginales de los cauces naturales de la Resolución Jefatural 332 - 2016 - ANA Reglamento para la Delimitación y Mantenimiento de Fajas Marginales, indica que en caso de resultar insuficiente el ancho mínimo es posible tener en consideración anchos de faja marginal de mayor longitud, bajo este criterio y debido a los resultados obtenidos en el modelamiento hidráulico y la vista de huellas máximas se ha asignado un ancho mínimo de $30 \mathrm{~m}$ de faja marginal.

\section{Conclusión}

Se realizó el modelamiento hidrológico para la quebrada palca al que al no contar con estaciones hidrométricas ni pluviométricas se generó empíricamente datos hidrométricos considerando que la quebrada Palca aporta caudal al Rio Caplina durante las épocas de máximas avenidas, Por lo tanto los caudales máximos instantáneos estimados para el modelamiento hidráulico son $6,37 \mathrm{~m}^{3} / \mathrm{s}, 12,33$ $\mathrm{m}^{3} / \mathrm{s}, 27,13 \mathrm{~m}^{3} / \mathrm{s}, 36,05 \mathrm{~m}^{3} / \mathrm{s}$ y $47,01 \mathrm{~m}^{3} / \mathrm{s}$ y $82,55 \mathrm{~m}^{3} / \mathrm{s}$ para periodos de retorno de $2,5,10,25,50$ y 100 años respectivamente. Para un periodo de retorno de 2 años el área de inundación será de 14,90m² en el margen derecho del cauce en el distrito de Calana, Para un periodo de retorno de 5 años el área de inundación será de $16,46 \mathrm{~m}^{2}$ en el margen derecho del cauce del distrito de Calana, Para un periodo de retorno de 10 años el área de inundación será de $09,09 \mathrm{~m}^{2}$ en el margen izquierdo del cauce del distrito de Pachía y $47,27 \mathrm{~m}^{2}$ en ambas márgenes del cauce en el distrito de Calana, Para un periodo de retorno de 25 años el área de inundación será de $80,15 \mathrm{~m}^{2}$ en el margen izquierdo del cauce del distrito de Pachía y 99,22m2 en el margen derecho del cauce en el distrito de Calana, Para un periodo de retorno de 50 años el área de inundación será de $190,6 \mathrm{~m} 2$ en el distrito de Pachía y $197,75 \mathrm{~m}^{2}$ en el margen derecho del cauce en el distrito de Calana, Para un periodo de retorno de 100 años el área de 
inundación será de $420,90 \mathrm{~m}^{2}$ en el margen izquierdo del distrito de Pachía y $252,93 \mathrm{~m}^{2}$ en el margen derecho del cauce en el distrito de Calana.

\section{Referencias Bibliográficas}

Pastrana, 2011, Modelamiento hidráulico del rio seco con fines de analizar la vulnerabilidad del distrito de Gregorio Albarracín ante caudales máximos, Tesis, Tacna,

Pino. 2013, Representación de un estudio hidro geomorfológica de la cuenca Caplina del departamento de Tacna, Tesis, Tacna,

Vilcanqui. 2016, Modelación hidrológica con fines de gestión de riberas en la cuenca del rio Caplina Tacna, Tesis, Tacna,

Ramírez. 2017, Propuesta metodológica para la delimitación de fajas marginales de los ríos, Investigación científica, Perú,

Resolución Jefatural №332, 2016, Reglamento para la Delimitación y Mantenimiento de Fajas Marginales de la Autoridad Nacional del Agua,

CENEPRED, 2015, Manual para la evaluación de riesgos originados por fenómenos naturales, 2da versión.

SINAGERD, 2014, Plan nacional de gestión del riesgo de desastres.

INDECI, 2011, Manual básico de estimación de riesgos, Manual, Perú. 